\title{
Connection-Oriented Routing in Ad Hoc Networks Based on Dynamic Group Infrastructure
}

\author{
Yu-Liang Chang, Ching-Chi Hsu \\ Department of Computer Science and Information Engineering, \\ National Taiwan University, Taiwan ROC. \\ d2506007@csie.ntu.edu.tw, cchsu@csie.ntu.edu.tw
}

\begin{abstract}
An ad hoc network is formed by a group of mobile hosts communicating over wireless channels. Such a network operates without any fixed network interaction and centralized administration. For the network environment is highly dynamic, the established routing paths are so fragile to maintain stable communication, especially the connection-oriented communications are quite difficult to be maintained. In our approach, the network infrastructure is initiall $3 y$ constructed by several communication groups, which are called Dynamic Group Infrastructure. This paper proposes the group-level connection maintenance based on Dynamic Group Infrastructure to implement connection-oriented routing. Our approach enhances the routing robustness of connection-oriented communications. The simulation results show that this routing approach under such construction outperforms the previous works in message complexity for connection-oriented communications.
\end{abstract}

\section{Introduction}

Mobile communication and wireless networking are becoming more and more popular. The mobile communication environment is often integrated into traditional networks as the Internet or Intranet $[1,7]$. An ad hoc network is temporarily formed by a group of mobile hosts communicating over wireless channels without any fixed network interaction and centralized administration. In this infrastructure all the mobile hosts communicate with other mobile hosts in wireless multihop routing style. All the mobile hosts act as routers in the network. Because the routers are mobile, the network topology is dynamic. The routing functions in ad hoc networks are more difficult and fragile than traditional networks. Recently there are many applications that require connection-oriented services in ad hoc networks, such as multimedia data communication. It is great challenge to perform connection-oriented routing in such dynamic network topology. It is important to develop an efficient routing scheme in ad hoc networks. Especially in connection-oriented communication the routing scheme should maintain effective and robust connections.

Recently there are researches on routing in ad hoc networks. P.Krishna et al. proposes clustering the ad hoc network as a two-level network graph $[11,16,17]$. Upper level is cluster-level connected graph. Lower level is the origin node-level connected graph. Z.J. Haas et al. proposes the zone routing scheme $[8,9,10]$, which is somewhat similar to cluster-based approach. For each mobile node it maintains all the links within the defined radius, so-called "zone". When route request is issued, it searches within the zone. Moreover the inter-zone searching is executed. B.Das et al. proposes another approach $[2,6]$. They use the characteristics of minimum connected dominating set to construct so-called virtual backbone in an ad hoc network. All the nodes not in the virtual backbone have their dominators that are the access points on the virtual backbone. A mobile host communicates with other mobile hosts may be routed through the virtual backbone by its dominator

In our approach [3], by comparing the information of connectivity with its neighbors, each mobile node determines itself as positive or non-positive node. After all the mobile nodes have determined their properties of the two types, all the connected positive nodes are grouped as a positive cluster and all the connected nonpositive nodes are grouped as a non-positive cluster. The two types of constructed clusters are interleaved with each other in an ad hoc network. The positive cluster plays the role of a topology information center for its adjacent nonpositive clusters. When a mobile node wants to communicate with other mobile nodes, it should at first perform route searching via local topology information. While the routes are not found, it issues route searching through the network. Because of the maintenance of topology information in positive clusters, the traditional path searching algorithms can be applied in the domain of a positive cluster and it adjacent non-positive clusters, we call the domain as a routing group. Routing in ad hoc networks is by means of routing group construction and maintenance.

For the frequent movement of mobile hosts in an ad hoc network, the found routes may be broken during connection-oriented communication. Under the constructed infrastructure, the broken route recovery is 
implemented by backtracking to the last routing group. The rerouting for the route proceeds in this routing group. This paper will show how it is done in our constructed infrastructure.

\section{Dynamic Group Infrastructure}

In this section, at first we describe the ad hoc network model and definitions for dynamic group routing. Some terminology is similar to the definition of IETF Internet draft [20]. Some other terminology is first defined in previous paper [3]. The dynamic group routing method is described later.

\subsection{Ad Hoc Network Model}

In a mobile ad hoc network, all the mobile hosts communicate with each other by wireless channels. A physical medium that can sustain data communication between two hosts is called a link. Both of the two hosts can communicate with each other by local broadcast. If there is a link between two hosts, we call that they are to be neighbors of each other. Each host in the network can detect its neighbors by local broadcast. Each host will broadcast beacon control signal in a time period such that it can detect the "connection" or "disconnection" of a link. The control signal may communicate over the dedicated control channels.

Messages are routed by multi-hop style in an ad hoc network. Each host acts as a router. They are assumed to be willing to forward the message when forwarding request is made. Moreover there is no cheating along these intermediate hosts when message is forwarding.

\subsection{Definitions}

The following definitions will be used to perform connection-oriented communication. For a mobile host $p$ in ad hoc networks, $\operatorname{deg}(p)$ is the degree of this host. In other words the host $\mathrm{p}$ has $\operatorname{deg}(p)$ neighbors. $\mathrm{N}(p)$ indicates the neighbors of host $p$.

Definition 2.1: $\mathrm{DV}(p)$ is the dominating value of a host $p$ in an ad hoc network. It is calculated as following before the construction proceeds.

$$
\begin{gathered}
\text { Initial } D V(p):=0 \\
\text { For each } q \text { which } q \text { in } N(p) \\
\text { If } \operatorname{deg}(p)>\operatorname{deg}(q) \text { then } \\
D V(p):=D V(p)+1 \\
\text { else if } \operatorname{deg}(p)<\operatorname{deg}(q) \text { then } \\
D V(p):=D V(p)-1
\end{gathered}
$$

When the routing groups are constructed initially, the dominating values of mobile hosts are used as the parameters to perform construction process. The dominating value $\mathrm{DV}(p)$ is got by comparing the bidirectional link connectivity of all neighbors. Initially $\operatorname{DV}(p)$ is zero. It compares with neighbors by degree values. If the degree of one of its neighbors is less than $\operatorname{deg}(p), \operatorname{DV}(p)$ is increased by one. Otherwise the neighbor's degree is greater than $\operatorname{deg}(p), \operatorname{DV}(p)$ is decreased by one.

After the construction process, an ad hoc network forms routing environment. There are some terminology defined in this paper.

Definition 2.2: In a constructed ad hoc network, the cluster formed by all connected positive nodes is called positive cluster (P-cluster). The cluster formed by all connected non-positive nodes is called non-positive cluster (N-cluster).

Definition 2.3: A routing group (RG) is formed by one $\mathrm{P}$ cluster with several attaching $\mathrm{N}$-clusters.

Definition 2.4: An attachable set is a set of all the positive nodes in the same P-cluster such that they have direct bi-directional links to the same adjacent $\mathrm{N}$-cluster.

Definition 2.5: A bridge cluster is the N-cluster belonged to more than one RG. On the other hand, the N-cluster belonged to one RG is called peripheral cluster.

In figure 1, the initial constructed network forms two routing groups $\mathrm{RG} 1$ and $\mathrm{RG} 2$. In routing group $\mathrm{RG} 1$ there are one $\mathrm{P}$-cluster and four $\mathrm{N}$-clusters. In routing group RG2 there are one P-CLUSTER and two N-clusters. The attachable set between P-cluster $\{\mathrm{D}, \mathrm{E}, \mathrm{H}, \mathrm{I}\}$ and $\mathrm{N}$ cluster $\{\mathrm{F}, \mathrm{G}\}$ is $\{\mathrm{E}, \mathrm{I}\}$. In other words The bridge cluster between RG1 and $R G 2$ is $\mathrm{N}$-cluster $\{\mathrm{M}, \mathrm{N}\} . \quad \mathrm{N}$ clusters $\{\mathrm{A}, \mathrm{B}, \mathrm{C}\},\{\mathrm{F}, \mathrm{G}\},\{\mathrm{J}, \mathrm{K}, \mathrm{L}\}$ and $\{\mathrm{P}, \mathrm{Q}, \mathrm{R}\}$ are all peripheral clusters.

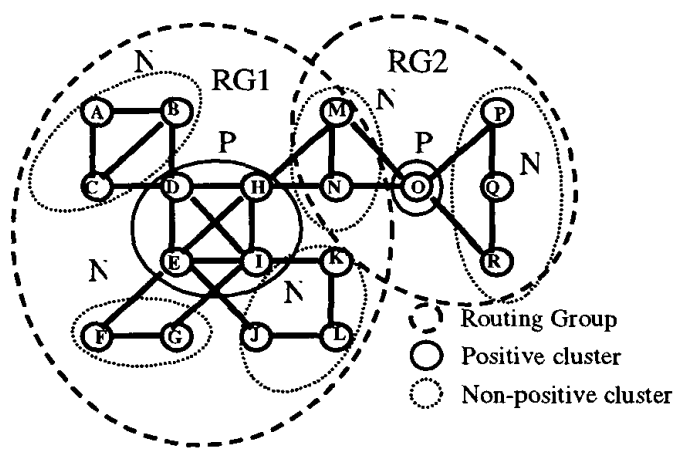

Figure 1. The initial constructed network topology

\section{Methodology of Dynamic Group Routing}

The procedures of group construction are developed to model the whole network into many routing groups [3]. In such constructed environment, route discovery is performed by the characteristics of routing groups. Later the network topology is changed due to some events of mobile nodes. The routes are maintained to apply the current network topology. Finally due to the dynamic of ad hoc network topology, the routing groups will react to the variations and update the current network topology. 


\subsubsection{Construction and Maintenance}

When several mobile nodes gather in some area, initially the network graph is not constructed. All the mobile nodes can only communicate with the nodes in its transmitting range. A mobile node senses the neighbors. In our approach the ad hoc mobile network will be constructed by some interactions with neighbors. A mobile node should sense the number of its neighbors. In this phase the plain information is the link connectivity for all the mobile nodes. With the connectivity information in neighborhood, the mobile node exchanges degree message with its neighbors. After gathering degrees of its neighbors, the mobile node calculates its dominating value and broadcasts the neighbor list with its dominating values. Upon receiving the messages of neighbor lists and dominating values, whether a mobile node propagates the message or not, it depends on the received dominating value and its dominating value. If the dominating value of receiver is positive, it propagates the message. While the dominating value of receiver is nonpositive and the received dominating value in nonpositive, it also propagates the message to its neighbors. The construction algorithm is derived as the following steps:

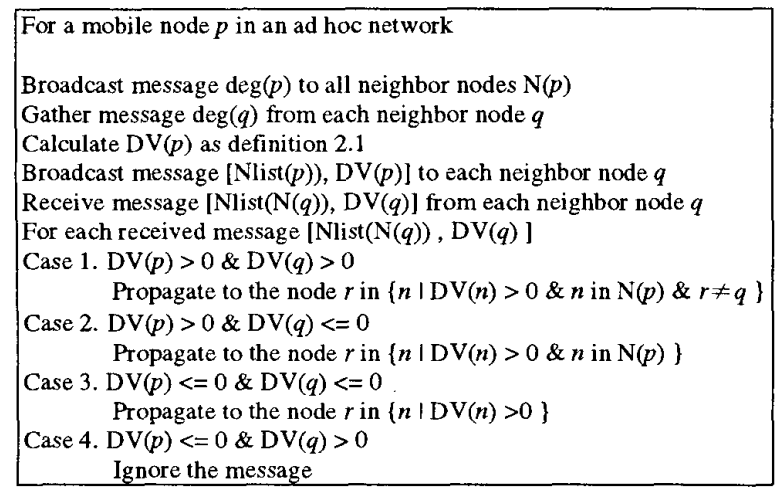

$\operatorname{Nlist}(q)$ is the data structure containing the neighbor identifiers of the mobile node $q$. Accomplishing the construction, the connected ad hoc network is modeled by two types of clusters: P-clusters and N-clusters. The network topology will be changed while some variant events occur in the network. The maintenance method is depicted in [3].

\subsubsection{Route Discovery Strategy}

Completeness of construction as section 2.3.1, the positive nodes keep the topology of its routing group. The P-cluster in a routing group plays the role of a topology information center for communication. When a mobile node wants to communicate to other nodes in this ad hoc network, at first it searches the routes in local information for intra-group routing. If the destination node belongs to this group, the routes are found. Otherwise it issues intergroup routing messages to all the nodes of the attachable sets the bridges clusters in this routing group. As the nonpositive source nodes the inter-group routing message will be propagated all over the ad hoc network. If the inter-group routes are found, they may be the style as figure 2 . From source nde $S$ to destination node $D$, there may be many attachable sets between them. It is important for an intermediate node to determine the forwarding attachable nodes for maintaining the found route in our method.

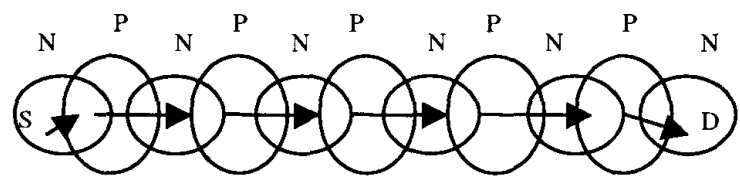

Figure 2. The found inter-group routes in the constructed ad-hoc network

\section{Connection-Oriented Routing via Dynamic Group Infrastructure}

In an ad hoc network all the nodes are mobile. The dynamic characteristics of nodes will affect the routing function in the network. In order to retaining the routing environment, the system should update the routing information by message passing through the network. Especially in case of the connection-oriented requirement the routes should be strongly sustained.

\subsection{Connection Setup in Ad Hoc Networks}

In the traditional networks the connection-oriented communication is performed on the higher layer than the network layer. In order to enhance the robustness and efficiency of the established connections in ad hoc networks, there is some difference to the routing in the traditional network. While the connection-oriented communication is initially requested on the higher layer, the connection setup is issued on the network layer and the related routing information will be kept in the intermediate nodes. The connection setup scenario is shown in figure 3. The source node sends a "ConnectQuery" packet to its attachable nodes in the same routing group. The intermediate nodes will cache the attachable node of the last routing group while the connection is across routing groups. The query packet is propagated to discover the route for the connection. When a route from the source node to the destination node is found, the "Connect-Report" packet is reply in the backward direction. The intermediate nodes also get the attachable node of the next routing group. The data structures of intermediate nodes are depicted in section 3.2. If the route fails to be found, it will receive "Connect-Fail" or 
wait for upper layer timeout.

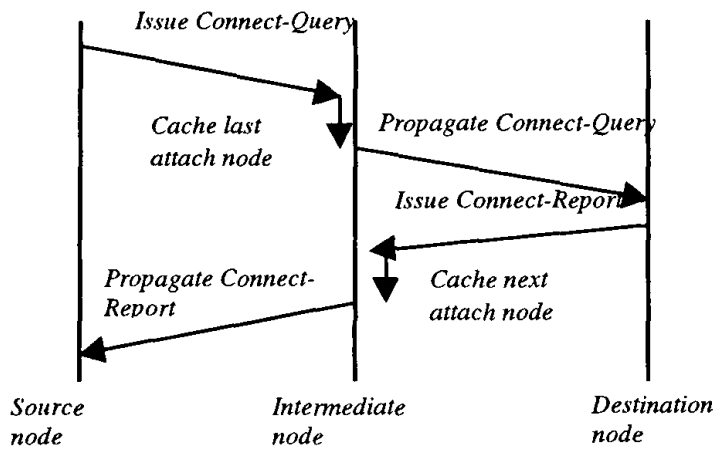

Figure 3. The scenario of connection setup in ad hoc networks

\subsection{Connection Information in An Intermediate Node}

When a connection is established, the intermediate nodes will record the related route information for such routes. For an intermediate node $a i$ on the established route its routing data structure is shown as figure 4 . "source" is the source node identifier. "destination" is the destination node identifier. "status" records the current routing states that is used in the routing mechanism. "backtrack" is the backtracking flag to determine the current process is normal routing or backtracking from the next attachable nodes. $a i-1$ is the identifier of the last node in this original found route. $a^{\prime} i-1$ is the identifier of the attachable node from ai to the last routing group. $a^{\prime} i$ is the identifier of the attachable node from the next routing to current routing group. $a^{\prime} i+1$ is the identifier of the attachable node from the current routing group to the next routing group. The information of the intermediate nodes will be used in connection-oriented recovery mechanism.

\begin{tabular}{|l|l|l|l|l|l|l|l|}
\hline Source & destination & status & backtrack & ai-1 & $a^{\prime} i-1$ & $a^{\prime} i$ & ai+1 \\
\hline
\end{tabular}

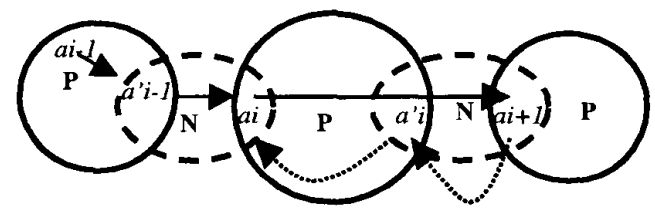

Figure 4. The information of the intermediate node a $i$ on an established connection

It is not necessary to record the whole nodes in an established route, because the dynamic group infrastructure maintains the local topology in the routing group. Moreover the network topology of an ad hoc network is dynamic. It is not reasonable to maintain the may-be-changed information in the near future. The intermediate nodes only keep the information of the access points to the last and the next routing groups. When packets are routed through the intermediate node, it can reach the attachable node of the outgoing group to forward the packets to the destination node.

\subsection{Connection-oriented Routing and Group-level Connection Maintenance}

In Dynamic Group infrastructure, the dynamic events are modeled as link variations of the mobile nodes. There are two states for a link between two nodes: connected or disconnected. A mobile node is switched on. It interacts with its nearby mobile nodes. When the connected state are detected by each other, the links between the switched-on node and its neighbors are constructed. If a mobile node is switched off, the links between the switched-off node and its neighbors are destroyed. In other words these links whose link states are changed into disconnected states. When a mobile node is moved from one place to another place, it causes several link variations including several disconnecting and connecting micro events. Such link variation occurs in neighborhood of some node. It will propagate the updating information to the non-positive nodes of the same $\mathrm{N}$-cluster and positive nodes of the same routing group. The connectionoriented routing strategies in the dynamic network environment are described as following.

Because all the nodes in attachable sets are positive nodes, they can easily detect the network topology in their routing group. In the routing process, there are several kinds of connection conditions from the intermediate node ai to its forwarding attachable node ai +1 :

Normal: the node ai+1 can be routed from node ai by the local topology information of routing group belonging to node ai.

Broken: the node ai+1 can't be routed from node ai by the local topology information of routing group belonging to node ai.

Merged: the node ai +1 retains positive, but it is in the same routing group of node ai.

Attached: the node ait 1 turns from positive to nonpositive.

The route recovery strategies will check the connection conditions of each ongoing path to next attachable nodes. If the detected connection condition is "Normal", the route process will proceed. Otherwise, the recovery process may be invoked. The recovery algorithm is shown as following: 


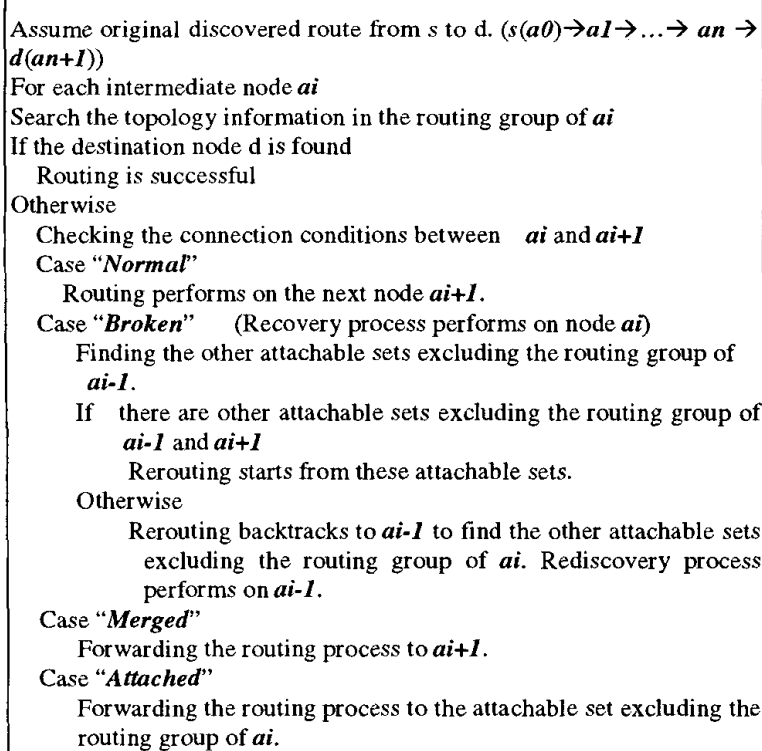

When the connection condition is "Broken", it backtracks to the node ai as figure 5. a'i finds attachable sets other than in the routing groups of ai-1. Therefore the rerouting is issued from the routing group. When the new partial route is discovered successfully in the attachable node a' $i+1$, it replies successful message to ai. ai updates the forwarding attachable set for the route. Otherwise it

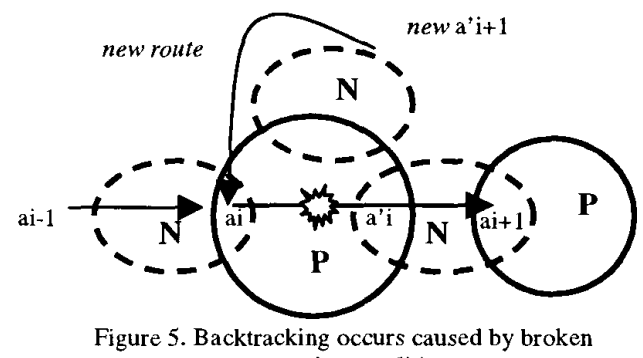

connection condition

backtracks to the source node. It reroutes for the connection. If it still fails, the disconnected is reported to the higher layer. The simulation results will show the performance on message complexity.

\section{Simulation Results}

The simulation model is that there are mobile hosts randomly distributed over a square area., for example , 800 meters *800meters. Each mobile host is with transmitting range, for example 200 meters. The communication range is the circle with radius of its transmitting range. There is a link between two mobile hosts while the distance of two mobile hosts is less than their transmitting range. A mobile host may be moving or pausing within a time click. It depends on the moving frequency. The maximum moving range is 20 meter within a time click. The connection-oriented calls are randomly chosen from two of the mobile nodes. During the connection-oriented call there is a packet delivered in a time click. We set a time click is 2 seconds and measure the ad hoc network execution in half an hour. In the execution time period the simulation result is shown as the following figures for variant mobility frequency and connection duration time. We focus on the performance of message complexity. We choose two different scale of ad hoc networks, 800meters*800meters with 32 mobile nodes and 1200 meters* 1200 meters with 72 mobile nodes. Figure 6.1 and figure 6.3 show the message complexity by different connection time duration. Figure 6.2 and figure 6.4 show the message complexity by different moving frequency of mobile nodes.

The existing routing schemes applied in the ad hoc network are classified either as proactive $[6,8,19,20]$ or as reactive $[12,13,14,18]$. Most of the routing schemes in an ad hoc network are hybrid of reactive and proactive schemes. In this paper, we compare with three hybrid schemes and our previous routing scheme that support somewhat fault tolerance in their infrastructures, Zone Routing approach $[8,9,10]$, MCDS(minimum connected dominating set) Routing approach $[2,6]$ and our Dynamic Group Routing approach[3], to compare to each other. For Zone Routing scheme, we choose radius equal to 2 .

Considering the connection-oriented communication, the established connections should be robust and not much message propagating overhead. The simulation results show that this scheme reduce the message complexity by keeping link states among routing groups and backtracking to the last routing group for rerouting. If the connection time duration increases, the reduction of message complexity is more obvious. Though this scheme may not choose the optimal shortest path in traditional routing scheme, it is suitable to massively reduce the message complexity in such all-mobile networks.

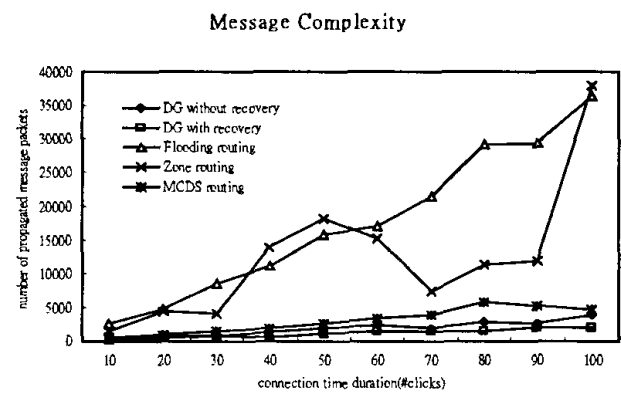

Figure 6.1 The message complexity comparison by connection time duration with area $=800 \mathrm{~m}^{*} 800 \mathrm{~m}$, number of mobile nodes $=32$, moving frequency $=50 \%$ 


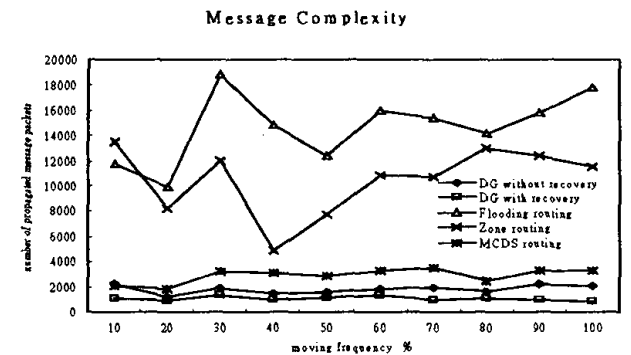

Figure 6.2 The message complexity comparison by mobile node moving frequency with area $=800 \mathrm{~m} * 800 \mathrm{~m}$, number of mobile nodes $=32$. connection duration $=50$

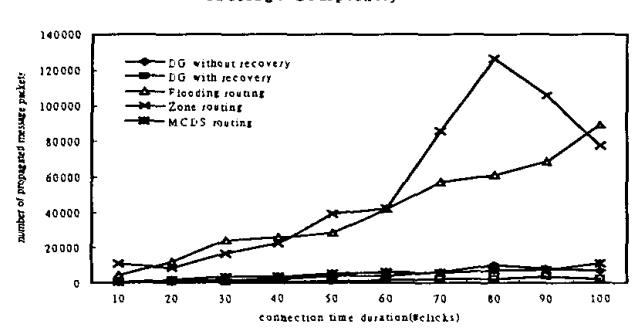

Figure 6.3 The message complexity comparison by connection time duration with area $=1200 \mathrm{~m}^{*} 1200 \mathrm{~m}$ number of mobile nodes $=72$, moving frequency $=50 \%$

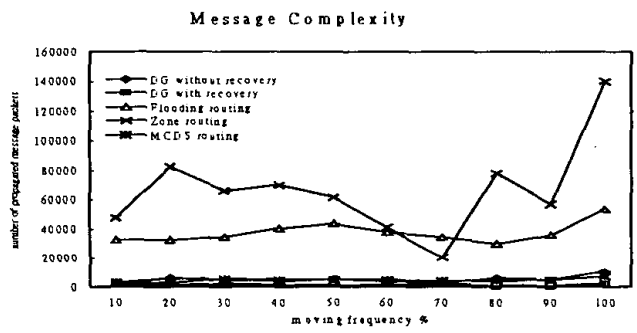

Figure 6.4 The message complexity comparison by mobile node moving frequency with area $=1200 \mathrm{~m} * 1200 \mathrm{~m}$, number of mobile nodes $=72$, connection duration $=50$

\section{Conclusion}

In order to improve the system performance in the realtime ad hoc network environment, the routing scheme should not too complicated to evaluate the volatile routes. In the connection-oriented requirement, the established routes should be maintained robustly and the messages should not massively propagate all over the network. In this paper, we propose a route recovery mechanism providing the efficient route recovery under the constructed dynamic routing group infrastructure. It seems that this approach is suitable to the connectionoriented communication while considering the message propagation overhead.. The furthermore steps for the research about routing in ad hoc network are developing protocols to manage the flow control all over the mobile ad hoc network and some other higher layer issues.

\section{References}

[1] P. Bhagwat, S.K. Tripathi, C. Perkins" Network Layer Mobility: an Arcitecture and Survey" IEEE Personal Communication , 3 (3), June 1996.

[2] V. Bharghavan and B. Das, "Routing in Ad Hoc Networks Using Minimum Connected Dominating Sets" IEEE International Conference on Communications 1997.

[3] Yu-Linag Chang \& Ching-Chi Hsu, "Routing in Wireless/Mobile Ad-hoc Networks via Dynamic Group Construction", to appear in ACM/Baltzer journal on Mobile Networks and Applications, 2000.

[4] M.S. Corson, A. Ephremides, " A Distributed Routing Algorithm for Mobile Wireless Networks" ACM Journal on Wireless Networks , 1995.

[5] M.S.Corson, J.Macker "Mobile Ad hoc Networking(MANET): Routing Protocol Performance Issues and Evaluation Considerations", Internet Draft, IETF,1997.

[6] B. Das, R. Sivakumar, and V. Bharghavan, "Routing in Ad-Hoc Networks Using a Spine" IEEE International Conference on Computers and Communications Networks 1997. [7] G. Forman, J. Zahorjan "The Challenges of Mobile Computing" Computer Science \& Engineering, University of Washington, US, IEEE Computer, Apr. 1994.

[8] Z.J.Haas \& M.R.Pearlman "The Zone Routing Protocol (ZRP) for Ad Hoc Networks", Internet Draft, IETF,1997.

[9] Z.J. Haas, "The Routing Algorithm for the Reconfigurable Wireless Networks" ICUPC'97, 1997.

[10] Z.J. Haas, "The Relaying Capability of the Reconfigurable Wireless Networks" VTC'97, 1997.

[11] M. Jiang, J. Li and Y.C. Tay, "Cluster Based Routing Protocol (CBRP) Functional Specification", Internet Draft , Internet Engineering Task Force, Mar. 1998.

[12] D.B. Johnson, "Scalable and Robust Internetwork Routing for Mobile Hosts" In Proceedings of the 14th International Conference on Distributed Computing Systems, IEEE Computer Society, Jun. 1994.

[13] D.B. Johnson, "Routing in Ad Hoc Networks of Mobile Hosts" IEEEE Proceedings of the Workshop on Mobile Computing Systems and Applications, pp. 158-163, Dec. 1994.

[14] D.B. Johnson and D.A. Maltz, "Dynamic Source Routing in Ad Hoc Wireless Networks". In Mobile Computing, edited by Tomasz Imielinski and Hank Korth, Chapter 5, pages 153-181, Kluwer Academic Publishers, 1996.

[15] D.Kim, S. Ha and Y. Choi, "K-hop Cluster-based Dynamic Source Routing in Wireless Ad-hoc Packet Radio Network", IEEE VTC'98, Ottawa, Canada, May 1998

[16] P.Krishna, N.H. Vaidya, D.K. Pradhan,"Static and Dynamic Location Management in Mobile Wireless Networks" Computer Communications, 1996.

[17] P.Krishna, N.H.Vaidya, M.Chatterjee, D.K.Pradhan, "A Cluster-Based Approach for Routing in Dynamic Networks"ACM SIGCOMM (CCR), 1997.

[18] C.Perkin, P. Bhagwat, "Highly Dynamic Destination Sequenced Distance Vector Routing (DSDV) for Mobile Computer" Proc. ACM SIGCOMM Symposium on Communication, Architectures and Protocol, pp. 234-244, 1994. [19] C.Perkins "Ad Hoc On Demand Distance Vector(AODV) Routing", Internet Draft, IETF, 1997.

[20] C.Perkins "Mobile Ad Hoc Networking Terminology", Internet Draft , IETF, Oct. 1997. 